\title{
Im „Namen der guten Sache“. Palästina-Solidarität, Antizionismus und Antisemitismus unter MuslimInnen im zeitgenössischen Frankreich
}

\author{
Alexandra Preitschopf
}

\section{Einleitung - die Pro-Palästina-Proteste im Sommer $2014^{1}$}

Anfang Juli 2014 reagierte Israel aufgrund verstärkten Raketenbeschusses durch die Hamas mit der Militäroffensive „Protective Edge“ im Gaza-Streifen. Dieser fielen auch viele palästinensische ZivilistInnen zum Opfer - nicht zuletzt aufgrund der Tatsache, dass die Hamas diese als Schutzschild benutzte - und in Reaktion hierauf fanden europaweit Proteste gegen das israelische Vorgehen statt, die in einigen Städten tausende Menschen mobilisieren konnten. ${ }^{2}$ Zwar verliefen diese oftmals friedlich, allerdings kam es immer wieder zu antisemitischen Manifestationen. ${ }^{3}$ Insbesondere in Frankreich war man auch mit gewalttätigen Ausschreitungen, bei denen mehrere Synagogen angegriffen und jüdische Geschäfte beschädigt wurden, konfrontiert. ${ }^{4}$ Am 13. Juli 2014 etwa blockierten mehrere Dutzend Demonstrierende eine Synagoge in Paris und hinderten die TeilnehmerInnen einer dort stattfindenden Zusammenkunft zur Unterstützung Israels bis zum Einschreiten der Polizei am Verlassen des Gebäudes. Mehrere Protestierende skandierten hierbei laut Medienberichten: „Tod den Juden! Hitler hatte Recht - wir werden euch abfackeln [...] Jihad! Jihad! Jihad! - Palästina muss die Arbeit [Hitlers] zu Ende bringen. "5 Im Pariser Vorort Sarcelles wiederum, wo eine der größten jüdischen Gemeinden Frankreichs lebt, wurden im Zuge einer nicht autorisierten Demonstration

1 Dieser Beitrag basiert auf meiner Dissertation: Alexandra Preitschopf, „Umkämpfter Raum“. Pro-PalästinaSolidarität, Antizionismus und Antisemitismus unter MuslimInnen im zeitgenössischen Frankreich, phil. Diss. Salzburg 2016.

2 Siehe zu Frankreich etwa Geoffroy Clavel, Manifestation pro-Palestine: un succès pour les organisateurs, malgré quelques dérapages isolés, in: Le Huffington Post, 24.7.2014,

[http://www.huffingtonpost.fr/2014/07/24/manifestation-pro-palestine-succes-organisateurs-derapages-

isoles_n_5615123.html], eingesehen 26.10.2018.

3 Siehe etwa Vladimir Vertlib, Der Tritt, in: Die Presse. Spectrum, 16.8.2014, S. III; Paul Lendvai, Judenhass gestern und heute, in: Der Standard, 5.8.2014, [http://derstandard.at/2000003981523/Judenhass-gestern-und-heute], eingesehen 26.10.2018;

4 Sylvie Corbet, Anti-Israel protesters clash with police outside Paris synagogue, in: Haaret\%, 13.7.2014, [https://www.haaretz.com/jewish/news/1.604879], eingesehen 26.10.2018

5 „Mort aux Juifs!- Hitler avait raison- On va vous cramer- [...] - Jihad ! Jihad ! Jihad !- La Palestine doit finir le boulot.“ (Zit. n. Frédéric Haziza, Israel-Palestine. Violences devant une synagogue à Paris: l'étrange réaction des médias, in: Le Nouvel Observateur, 15.7.2014, [http://leplus.nouvelobs.com/contribution/1223785-israel-palestine-violences-devantune-synagogue-a-paris-l-etrange-reaction-des-medias.html], eingesehen 26.10.2018. 
am 20. Juli u. a. eine jüdische Apotheke und ein koscherer Supermarkt ausgebrannt. ${ }^{6}$ Zuvor hatten Demonstrierende Jüdinnen und Juden vor einer Synagoge mit den Worten „Wir werden euch in Schutt und Asche legen“ bedroht. ${ }^{7}$ Jenseits der Demonstrationen wurden - allein im Monat Juli - in Frankreich insgesamt 201 antisemitische Vorfälle gemeldet, davon 61 Gewalttaten. ${ }^{8}$

Es stellt sich die schwierig $\mathrm{zu}$ beantwortende Frage, inwiefern diese Gewaltausschreitungen im Zuge mancher Proteste die Taten Einzelner blieben oder möglicherweise als repräsentativ für das Verhalten des Großteils der Demonstrierenden angesehen werden können. Zwar wurde von Seiten der Protest-Organisatoren sowie von zahlreichen Demonstrierenden immer wieder betont, nicht antisemitisch zu sein, sondern nur für die Rechte der PalästinenserInnen und die Beendigung der israelischen Militäroffensive eintreten zu wollen, ${ }^{9}$ nichtsdestotrotz waren die auf den Demonstrationen skandierten Parolen nicht immer unproblematisch. Neben der Solidaritätsparole „Nous sommes tous des Palestiniens“ („Wir sind alle Palästinenser“) wurde vielfach „Israël assassin, Hollande complice!“ („Mörder Israel, Komplize Hollande“) oder „Sionistes fascistes, c'est vous les terroristes“ (,Zionisten Faschisten, ihr seid die Terroristen“) skandiert, bis hin zu „Mort aux juifs“ („Tod den Juden“). Hinzu kamen Slogans wie „Nous sommes là pour lutter contre le nouveau nazisme“ („Wir sind hier um gegen den neuen Nazismus zu kämpfen“). Auf manchen Schildern war dementsprechend auch „Sionisme = nazisme“ zu lesen oder es wurden Hakenkreuz und Davidstern unmittelbar nebeneinandergestellt und mit einem Gleichheitszeichen verbunden. ${ }^{10}$ Manche Demonstrierende zeigten zudem den so genannten quenelle-Gruß, der auf den umstrittenen antisemitischen Komiker Dieudonné M'Bala M'Bala zurückgeht und von manchen französischen SozialwissenschaftlerInnen unter anderem als „umgekehrter Nazi-Gruß“" gedeutet wird. ${ }^{11}$

6 A Sarcelles, des dégradations après des manifestations interdites, in: Le Monde, 21.7.2014,

[http://www.lemonde.fr/societe/article/2014/07/20/a-sarcelles-des-degradations-apres-une-manifestationinterdite_4460283_3224.html]., eingesehen 26.10.2018.

${ }^{7}$ „On va vous mettre à feu et à sang“. (Zit. n. Anne Jouan, À Sarcelles: „Tu leur montres une carte, ils ne savent même pas où est la Palestine!“, in: Le Figaro, 21.7.2014, [http://www.lefigaro.fr/actualite-france/2014/07/21/0101620140721ARTFIG00348--sarcelles-tu-leur-montres-une-carte-ils-ne-savent-meme-pas-o-est-la-palestine.php], eingesehen 26.10.2018.)

8 Insgesamt registrierte das Service de Protection de la Communauté Juive, welches in Zusammenarbeit mit dem französischen Innenministerium antisemitische Vorfälle in Frankreich erfasst, für das erste Halbjahr 2014 insgesamt 326 Vorfälle und damit doppelt so viele wie im Zeitraum des Vorjahres. (Floriane Hohenberg/Ronald Eissens/Suzette Bronkhorst, Antisemitic Incidents in Europe before, during and after the Israel-Gaza conflict. A Report by The International Network Against Cyber Hate (INACH) and the Ligue Internationale contre le Racisme et l'Antisémitisme (LICRA), Amsterdam-Paris 2014, S. 10.)

${ }_{9}$ Mehdi Thomas Allal, Manif pro-Palestine: oui, on peut soutenir Gaza et critiquer Israël sans être antisémite, in: Le Nouvel Observateur, 26.7.2014, [http://leplus.nouvelobs.com/contribution/1227067-manif-pro-palestine-oui-on-peutsoutenir-gaza-et-critiquer-israel-sans-etre-antisemite.html], eingesehen 26.10.2018.

10 Nicolas Guégan, Manifestation pro-palestinienne: Nous sommes tous des Palestiniens, in: Le Point, 3.8.2014, [http://www.lepoint.fr/societe/manifestation-pro-palestinienne-nous-sommes-tous-des-palestiniens-03-08-20141851144_23.php], eingesehen 26.10.2018.

11 Nicolas Guégan, Affaire Dieudonné - Meyer Habib: La quenelle est le nouveau salut nazi, in: Le Point, 2.1.2014, 
Die Bandbreite an Parolen, Anspielungen auf Dieudonné bis hin zu antisemitischen Übergriffen gibt einen ersten Hinweis auf die heterogene Zusammensetzung der Demonstrierenden wie auch der OrganisatorInnen und der an den Demonstrationen teilnehmenden Gruppierungen: So waren zum einen linke bis linksradikale Organisationen und dezidiert pro-palästinensische Vereinigungen beteiligt, zum anderen nahmen zahlreiche Einzelpersonen ohne Zugehörigkeit zu einer bestimmten Organisation teil, darunter auch viele Demonstrierende mit muslimischem Hintergrund. ${ }^{12}$ Während der Protestzüge kam es immer wieder auch zu gemeinsamen Gebeten, manche Frauen demonstrierten im „Niqab“, auch Parolen wie ,allāhu akbar“ („Gott ist groß“) und vereinzelt „Djihad résistance“ waren zu hören. Dieser religiöse Aspekt der Proteste wurde zudem durch die Anwesenheit radikal-islamistischer und mitunter Hamas-naher Gruppierungen, wie das „Collectif Cheikh Yassine“, bestätigt insgesamt waren diese zahlenmäßig allerdings in einer deutlichen Minderheit. ${ }^{13}$ Die Präsenz radikal-islamistischer Vereinigungen auf Pro-Palästina-Demonstrationen lässt sich, dem französischen Politologen und Islamismus-Experten Jean-Yves Camus zufolge, in Frankreich vor allem seit der israelisch-palästinensischen Auseinandersetzung 2009 (Militäroperation „Gegossenes Blei“) und den damit verbundenen Protestveranstaltungen beobachten. ${ }^{14}$

\section{Ausgangssituation - innergesellschaftliche Spannungen und die Problematik des „neuen“ Antisemitismus im zeitgenössischen Frankreich}

Auch wenn sich im Nachhinein kaum näher bestimmen lässt, inwiefern die individuellen TeilnehmerInnen an den verschiedenen Pro-Palästina-Demonstrationen antizionistische und in manchen Fällen antisemitische Einstellungen vertraten, so stellt der Umstand, dass viele politisch eher der (radikalen) Linken zuzurechnen waren oder oftmals einen muslimischen Hintergrund hatten, doch einen wichtigen Anhaltspunkt hierfür dar. Diesbezüglich interessant sind auch die Ergebnisse einer aktuellen Studie zu antisemitischen Einstellungen in der französischen Gesellschaft von November 2014. Im Zuge der von der französischen Einrichtung Fondapol („Fondation pour l'innovation politique“, „Stiftung für politische Innovation“) durchgeführten Untersuchung waren

[http://www.lepoint.fr/societe/affaire-dieudonne-meyer-habib-la-quenelle-est-le-nouveau-salut-nazi-02-01-20141776259_23.php], eingesehen 26.10.2018.

12 Bernard Edinger, A worried community, in: The Jerusalem Report, 25.8.2014, S. 29.

13 Clément Parrot, Qui sont les participants aux manifestations pro-palestiniennes interdites?, in: franceinfo, 31.7.2014, [http://www.francetvinfo.fr/societe/manifestations-propalestiniennes-en-france/qui-sont-les-participants-auxmanifestations-pro-palestiniennes-interdites_658399.html], eingesehen 26.10.2018.

${ }_{14}$ Jean-Yves Camus, The French Extreme-Right, Anti-Semitism, and Anti-Zionism (1945-2009), in: Holocaust, Study and Research (Holocaust, Studii și cercetări) 5 (2012), S. 175-189, hier S. 182. 
zum einen 1.500 Personen als repräsentative Stichprobe für die französische Bevölkerung über 16 Jahre befragt worden, zum anderen als Vergleichsgruppe 575 Personen mit muslimischem Hintergrund (d. h. Personen, die angegeben hatten, in einer muslimischen Familie geboren worden zu sein). Bei den Befragungen war zudem ermittelt worden, welchem politischen Lager die jeweiligen Personen sich selbst zuordneten. Angesichts der erhobenen Datenlage zeichnen sich anhand der Ergebnisse der Studie Tendenzen ab, die sich in Frankreich bereits seit dem Jahr 2000 beobachten lassen: So war die Zustimmungsrate zu Items mit antizionistischen und antisemitischen Aussagen (1.) unter mit dem Front National sympathisierenden und Marine Le Pen wählenden Befragten erhöht, (2.) unter solchen, die sich der Front de gauche (eine Koalition verschiedener linksradikal, kommunistisch und antikapitalistisch geprägter Parteien) zuordneten sowie (3.) unter den befragten muslimischen Personen. Im Falle letzterer war zwischen Personen „muslimischer Herkunft“, „gläubigen MuslimInnen“ und „gläubigen und praktizierenden MuslimInnen“ unterschieden worden, wobei gläubige und zugleich praktizierende MuslimInnen antisemitischen Aussagen durchschnittlich stärker zustimmten als die beiden anderen Gruppen. ${ }^{15}$

Die genannte Studie gibt, wie bereits angedeutet, auch einen wichtigen Hinweis auf die generelle Problematik des ,neuen“ Antisemitismus, der in Frankreich verstärkt seit dem Jahr 2000 und dem Ausbruch der Zweiten Intifada in Israel zu beobachten ist. ${ }^{16}$ Manifest wurde dieser „neue“ Antisemitismus anhand einer deutlichen Zunahme und nach wie vor sehr hohen Zahl an antisemitischen Vorfällen, welche von Beschimpfungen, Schmierereien, Sachbeschädigungen bis hin zu Brandanschlägen auf Synagogen und andere jüdische Einrichtungen sowie tätlichen Übergriffen reichen. ${ }^{17}$ Traurige Kulminationspunkte waren hierbei drei islamistisch-jihadistisch motivierte Mordanschläge: Im März 2012 tötete der französische Jihadist Mohamed Merah drei jüdische Kinder und einen Lehrer in einer jüdischen Schule in Toulouse. Im Mai 2014 erschoss der französische Islamist Mehdi Nemmouche vier Menschen jüdischer Herkunft im Jüdischen Museum von Brüssel, im Januar 2015 tötete der sich zum Islamischen Staat bekennende Franzose Amedy Coulibaly im Zuge einer Geiselnahme

\footnotetext{
${ }^{15}$ Dominique Reynié, L'antisémitisme dans l'opinion publique française. Nouveaux éclairages, Paris 2014. - So hielten etwa 74\% der MuslimInnen die sich selbst als ,gläubig und praktizierend“ definierten, die Aussage, ,,die Juden“ hätten zu viel Macht und Einfluss im Wirtschafts- und Finanzbereich für zutreffend, im Gegensatz zu 52\% der befragten Personen „muslimischer Herkunft“ und $25 \%$ der befragten RepräsentantInnen der französischen Mehrheitsbevölkerung. (Ebd., S. 22.)

${ }^{16}$ Hierzu erschien ab dem Jahr 2001 eine Reihe an Publikationen. Siehe u. a. Raphaël Draï, Sous le signe de Sion. L'antisémitisme nouveau est arrivé, Paris 2001; Pierre-André Taguieff, La Nouvelle Judéophobie, Paris 2002; Alain Finkielkraut, Au nom de l'Autre. Réflexions sur l'antisémitisme qui vient, Paris 2003; Nicolas Weill, La République et les antisémites, Paris 2004; Michel Wieviorka, La tentation antisémite. Haine des Juifs dans la France d'aujourd'hui, Paris 2005; Ders., L'antisémitisme est-il de retour?, Paris 2008; Didier Lapeyronnie, Ghetto urbain. Ségrégation, violence, pauvreté en France aujourd'hui, Paris 2008.

17 Siehe u. a. Commission nationale consultative des droits de l'homme, La lutte contre le racisme, l'antisémitisme et la xénophobie. Année 2015. Les essentiels, Paris 2016, S. 6; Alexis Rosenbaum, L'antisémitisme, Levallois-Perret 2014, S. 8-9.
} 
vier jüdische Kunden eines koscheren Supermarkts in Paris. In zwei Fällen spielte auch die Bezugnahme auf Palästina eine nicht unwesentliche Rolle - Merah „rechtfertigte“ seine Tat damit, er hätte palästinensische Kinder rächen wollen, Coulibaly argumentierte ähnlich, er hätte „die unterdrückten Muslime in der Welt“, vor allem in Palästina, rächen wollen. ${ }^{18}$

Diese Denkweise, die jüdische Bevölkerung Frankreichs kollektiv und unmittelbar mit Israel zu identifizieren und in weiterer Folge als „feindlich“ zu betrachten, ist stark in islamistischen Milieus ausgeprägt, findet sich jedoch auch in weiteren sozialen und politischen Milieus. Generell ist im medialen und politischen Diskurs Frankreichs immer wieder die Rede von einem „Import des Nahostkonflikts“, welcher sich in „,interkommunitäre Spannungen“ zwischen der muslimischen und jüdischen Bevölkerung übersetze. ${ }^{19}$ Hierbei wird die Brisanz der Situation in demographischer Hinsicht auch durch den Umstand verstärkt, dass in Frankreich heute sowohl eine der größten muslimischen Bevölkerungsgruppen (fünf bis sechs Millionen MuslimInnen bzw. acht bis zehn Prozent der Bevölkerung) als auch die größte jüdische Bevölkerungsgruppe (etwa 500.000 Jüdinnen und Juden) in Europa lebt. ${ }^{20}$

Allerdings sind Solidarisierungs- und Identifikationsprozesse und mitunter damit einhergehende antisemitische Manifestationen unter MuslimInnen in Frankreich nicht nur im Kontext des Nahostkonflikts zu betrachten, sondern stehen auch in Zusammenhang mit innerfranzösischen Entwicklungen. Dies betrifft im Besonderen die französische Erinnerungspolitik und den Umgang des französischen Staates mit der eigenen Vergangenheit: Herrschte in Frankreich nach 1945 zunächst weitgehendes Schweigen über die Shoah und die Mitverantwortung des Vichy-Regimes, wurde diese „Phase der Verdrängung“ (H. Rousso) erst nach und nach von einer zögerlichen Aufarbeitung abgelöst - eine Aufarbeitung, welche sich heute in einer umfassenden und institutionalisierten Gedenkkultur manifestiert. ${ }^{21}$ Damit einhergehend ist in den letzten Jahren jedoch auch - im Zuge der stärkeren Differenzierung und „Ethnisierung“ der französischen Gesellschaft - das Phänomen einer so genannten „concurrence

18 Siehe u. a. Caroline Politi/Eric Pelletier, Merah, Kouachi, Coulibaly... D’incroyables similitudes, in: L'Express, 14.1.2015, [http://www.lexpress.fr/actualite/societe/merah-kouachi-coulibaly-d-incroyables-similitudes1640392.html], eingesehen 26.10.2018; Eric Hazan, Massacre antijuif de Vincennes: le terroriste a agi pour venger les Palestiniens, in: Le Monde juif, 9.1.2015, [https://www.lemondejuif.info/2015/01/massacre-antijuif-vincennesterroriste-agi-venger-les-palestiniens], eingesehen 26.10.2018.

${ }^{19}$ Siehe u. a. Sylvain Attal, Aux racines du nouvel antisémitisme, in: Revue internationale et stratégique 58 (2005), Heft 2, S. 57-66, hier S. 57; Eric Keslassy, Présentation. „Sociologiser“ le Proche-Orient pour renforcer la République, in: Frédéric Encel/Eric Keslassy (Hrsg.), Comprendre le Proche-Orient. Une nécessité pour la République, Paris 2005, S. 17-36, hier S. 30.

20 Siehe bzgl. dieser Problematik u. a. die Berichte zu den Jahren 2004 bis 2015 der Commission nationale consultative des droits de l'homme, La lutte contre le racisme, l'antisémitisme et la xénophobie (Paris 2005-2016) sowie Wolfram Stender, Konstellationen des Antisemitismus, in: Wolfram Stender/Guido Follert/Mihri Özdogan (Hrsg.), Konstellationen des Antisemitismus. Antisemitismusforschung und sozialpädagogische Praxis, Wiesbaden 2010, S. 738, hier S. 27; Danny Leder, Die Gefahr aus der Vorstadt. Bei Frankreichs Migrantenjugend greift altneuer Judenhass, in: Das Jüdische Echo 55 (2006), S. 135-145.

${ }^{21}$ Siehe exemplarisch Weill, La République et les antisémites, S. 13. 
mémorielle“, einer „Erinnerungskonkurrenz“ zu beobachten. So orientieren sich in Frankreich zunehmend Angehörige weiterer Minderheiten am Modell des nationalen Shoah-Gedächtnisses, um auf diese Weise die öffentliche Anerkennung „eigener“ historischer Traumata (beispielsweise im Kontext des Algerienkrieges, des französischen Kolonialismus oder der Sklaverei) ${ }^{22}$ einzufordern. ${ }^{23}$ Die öffentliche Thematisierung und Anerkennung wie auch das offizielle Gedenken an diese anderen Formen historischen Unrechts setzte ohnehin erst sehr verspätet ab den 2000er-Jahren ein. All dies wirkt sich auf die Wahrnehmung und die Einstellung zur jüdischen Bevölkerung Frankreichs aus, deren (scheinbar) „vorrangiger Opferstatus“ zum Teil im Sinne einer Opferkonkurrenz mit Ressentiments beargwöhnt wird. Dies artikuliert sich mitunter in einer Relativierung der Shoah, in Gleichsetzungen von nationalsozialistischer und israelischer Politik (wie in den eingangs erwähnten Demonstrationsparolen) oder in einer Delegitimierung Israels als „neue (rassistische) Kolonialmacht“. ${ }^{24}$ Werden die PalästinenserInnen auf diese Weise $\mathrm{zu}$ „den neuen Kolonisierten“ stilisiert, bietet dies wiederum für französische MuslimInnen mit maghrebinischem oder subsaharisch-afrikanischem Hintergrund insofern eine Identifikationsmöglichkeit, da deren eigene Vorfahren oftmals tatsächlich kolonisiert worden waren.

Jenseits der Nachwirkungen der französischen Kolonialvergangenheit und dem Konflikt um die französische Erinnerungspolitik sind weitere soziopolitische Entwicklungen in den Blick zu nehmen: Der Soziologe Didier Lapeyronnie etwa betrachtet antisemitische Manifestationen unter muslimischen Jugendlichen aus sozial prekären Banlieues französischer Großstädte vor allem als eine Ausdrucksform des städtischen „Ghettos“ und deutet diese als Folgewirkung verstärkter innergesellschaftlicher Rückzugs- und Abgrenzungstendenzen. ${ }^{25}$ So lässt sich in Frankreich - gerade in multiethnisch geprägten Vororten - seit einigen Jahren vermehrt ein so genannter „repli communautaire“, das heißt ein stärkerer Rückzug ethnischer oder religiöser Gemeinschaften auf sich selbst beobachten, ${ }^{26}$ der auch für den muslimisch-jüdischen Dialog ein Hindernis darstellt. ${ }^{27}$ Eine zum Teil ähnliche Argumentation verfolgt die französische Historikerin Esther

22 Sven Korzilius, Erinnerungsforderungen von descendants d'esclaves - Berechtigtes Anliegen oder Missbrauch der Geschichte?, in: Dietmar Hüser (Hrsg.), Frankreichs Empire schlägt zurück. Gesellschaftswandel, Kolonialdebatten und Migrationskulturen im frühen 21. Jahrhundert, Kassel 2010, S. 217-252.

${ }^{23}$ Siehe u. a. Pascal Blanchard/Isabelle Veyrat-Masson (Hrsg.), Les Guerres de mémoires. La France et son histoire. Enjeux politiques, controverses historiques, stratégies médiatiques, Paris 2008; Geoffrey Grandjean/Jérôme Jamin, La concurrence mémorielle, Paris 2011.

${ }^{24}$ Siehe exemplarisch Rosenbaum, L'antisémitisme, S. 60-61.

${ }^{25}$ Lapeyronnie, Ghetto urbain, S. 395.

${ }^{26}$ Siehe u. a. Mohand Khellil, Maghrébins de France. De 1960 à nos jours: la naissance d'une communauté, Toulouse 2004, S. 101-104; Dietmar Hüser, Plurales Frankreich in der unteilbaren Republik. Einwürfe und Auswüchse zwischen Vorstadt-Krawallen und Kolonial-Debatten, in: Deutsch-Französisches Institut (Hrsg.), Frankreich Jahrbuch 2006. Politik und Kommunikation, Wiesbaden 2007, S. 9-28, hier S. 23; Vincent Geisser/Aziz Zemouri, Marianne et Allah. Les politiques français face à la „question musulmane“, Paris 2007, S. 183-184.

${ }_{27}$ Siehe u. a. Gilles Kepel, Quatre-vingt-treize. Essai, Paris 2012, S. 95-96; Daniel Bensoussan-Bursztein, Étude 2011. Le dialogue judéo-musulman en France d'hier à aujourd'hui, in: Regards. Revue du Centre Communautaire Lä̈ Juif de Bruxelles. Etude annuelle de Regards (2011), S. 1-23. 
Im „Namen der guten Sache“

Benbassa, die in diesen Ethnisierungs- und Re-Religiösierungs-Tendenzen einen Ausdruck des Scheiterns des französischen Integrationsmodells sieht. Insbesondere unter einigen muslimischen Jugendlichen sei der „,nationalen Identität“ inzwischen eine „Diaspora-Identität“ und ein Gefühl der globalen Verbundenheit im Sinne einer transnationalen Religionsgemeinschaft (der „Umma“) entgegengetreten. Hierbei werde wiederum die ideelle Solidarisierung mit „muslimischen Brüdern und Schwestern“, insbesondere den PalästinenserInnen, zu einem wesentlichen identitätsstiftenden Faktor. $^{28}$

Angesichts dieser Diagnosen, die einen Verlust der Attraktivität des französischen Integrationsmodells konstatieren, stellt sich umso mehr die Frage, ab wann und inwiefern Palästina speziell für MuslimInnen in Frankreich eine wichtige identitätspolitische Rolle zu spielen begann.

\section{Pro-palästinensische „Tradition“ seit 1967 und neue Formen der Palästina-Solidarität}

Erste tiefgreifende Auswirkungen hatte in dieser Hinsicht der Widerhall des Sechstagekriegs von 1967 in Frankreich. Während Israel für französische Jüdinnen und Juden ab diesem Zeitpunkt zunehmend an Bedeutung gewann, ${ }^{29}$ versuchten andererseits Gruppierungen der radikalen Linken an französischen Universitäten und unter nordafrikanisch-muslimischen Immigranten für die Solidarität mit Palästina zu mobilisieren. ${ }^{30}$ Anfang der 1970er-Jahre nahmen diese Versuche noch weiter an Intensität zu, als von der maoistischen Gauche prolétarienne („Arbeiterlinke“) so genannte „Comités de soutien à la révolution palestinienne“ („Unterstützungskomitees für die palästinensische Revolution“) gegründet wurden, die nicht nur für die Rechte der PalästinenserInnen eintraten, sondern zugleich für jene der maghrebinischen Arbeiter in Frankreich. ${ }^{31}$ Die aus französischen Studierenden und arabischen Arbeitern zusammengesetzten Komitees positionierten sich sowohl für Palästina als auch gegen Rassismus und für eine Verbesserung der Lebensbedingungen der maghrebinischen Arbeitsimmigranten. ${ }^{32}$ Palästina-Solidarität im Frankreich der späten 1960er und frühen 1970er-Jahre ist somit weniger als Manifestation „muslimisch-jüdischer“ Divergenzen, sondern eher im Kontext eines linken Antizionismus zu sehen, der sich in dieser Zeit

${ }^{28}$ Esther Benbassa, Juifs et Musulmans: le modèle républicain renégocié, in: Mouvements 38 (2005), S. 60-67, hier S. 60. ${ }^{29}$ Esther Benbassa, Frankreich, in: Elke-Vera Kotowski/Julius H. Schoeps/Hiltrud Wallenborn (Hrsg.), Handbuch zur Geschichte der Juden in Europa. Band 1. Länder und Regionen, Darmstadt 2001, S. 387-418, hier S. 415.

${ }^{30}$ Maud S. Mandel, Muslims and Jews in France. History of a conflict, Princeton 2014, S. 98-101.

31 Olivia C. Harrison, Performing Palestine in Contemporary France: Mohamed Rouabhi's Transcolonial Banlieue, in: Modern \& Contemporary France 22 (2014), Heft 1, S. 43-57, hier S. 45-46.

32 Abdellali Hajjat, Les comités Palestine (1970-1972). Aux origines du soutien de la cause palestinienne en France, in: Revue d'études palestiniennes (2005), S. 9-27, hier S. 10. 
nicht nur in Frankreich innerhalb des Kampfes der radikalen Linken gegen „Imperialismus“, Kolonialismus und für „Dritte-Welt-Aktivismus“ ausbildete. ${ }^{33}$

In der maghrebinischen beziehungsweise muslimischen MigrantInnen-Community in Frankeich bestand Ende der 1960er-Jahre hingegen nur geringes Interesse für die „palästinensische Sache“ - trotz der Aufrufe und Mobilisierungsversuche, die an sie gerichtet wurde. ${ }^{34}$ Hierbei sollte jedoch bedacht werden, dass von MuslimInnen ausgehende politische Aktivitäten bis in die 1970er-Jahre ohnehin eher ein Randphänomen darstellten und dies schon allein dadurch bedingt war, dass die strukturellen Voraussetzungen hierfür weitgehend fehlten und die Mehrzahl erst im Laufe der 1960er-Jahre eingewandert war. Die Bedingungen änderten sich zu Beginn der 1980er-Jahre, als eine neue Generation an bereits in Frankreich geborenen jungen MuslimInnen hör- und sichtbarer wurde. Diese so genannte Beur ${ }^{35}$-Bewegung entstand im Kontext eines gesellschaftspolitischen Klimas, in welchem es, wie bereits erwähnt, zu einer schärferen religiösen und ethnischen Differenzierung der Gesellschaft kam, zugleich jedoch zu einem Anstieg an rassistischen Straftaten und zum Aufstieg der extremen Rechten, insbesondere des Front National. ${ }^{36}$ In Reaktion hierauf wurden einerseits mehrere antirassistische Organisationen (bspw. 1984 „SOS Racisme“) gegründet, die sich dem gemeinsamen Kampf gegen Rassismus und Antisemitismus verschrieben. ${ }^{37}$ Andererseits wurde die Solidarisierung mit dem Schicksal der PalästinenserInnen gerade für junge französische MuslimInnen zu einer Ausdrucksmöglichkeit von Frust und Unzufriedenheit, führte jedoch zugleich zu einer stärkeren Abgrenzung gegenüber der jüdischen Bevölkerung. ${ }^{38}$ Dies verstärkte sich insbesondere mit dem Libanonkrieg 1982, welcher politische Bruchlinien zwischen der jüdischen und muslimischen Bevölkerung in Frankreich erstmals umfassender deutlicher zu Tage treten ließ.39 Junge „Beur-AktivistInnen“ brachten ihre Unterstützung für Palästina vehement zum Ausdruck, wobei mitunter auch antisemitische Parolen wie ,jüdische Mörder“ und „Tod den Juden“ sowie verschwörungstheoretische Diktionen von der ,jüdischen Macht“ oder dem , jüdischen Geld“ zu hören waren. ${ }^{40}$

\footnotetext{
33 Siehe u. a. Michel Winock, La France et les Juifs. De 1789 à nos jours, Paris 2004, S. 319; Helga Embacher, Neuer Antisemitismus in Europa - Historisch vergleichende Überlegungen, in: Moshe Zuckermann (Hrsg.), Antisemitismus - Antizionismus - Israelkritik (Tel Aviver Jahrbuch für deutsche Geschichte 33), Göttingen 2005, S. 50-69, hier S. 65; Martin Kloke, Linker Antisemitismus, in: Wolfgang Benz (Hrsg.), Handbuch des Antisemitismus. Judenfeindschaft in Geschichte und Gegenwart. Band 3: Begriffe, Theorien, Ideologien, Berlin-New York 2010, S. 192-195, hier S. 193.

34 Siehe u. a. Mandel, Muslims and Jews, S. 111; Hajjat, Les comités Palestine, S. 10.

35 „Beur“ ist ein umgangssprachlicher Ausdruck für frz. „Arabe“ (dt. „Araber“).

${ }^{36}$ Mandel, Muslims and Jews, S. 128.

${ }^{37}$ Ebd., S. 136.

38 Diese Spannungen brachte, als einer der Ersten, der französisch-jüdische Intellektuelle Alain Finkielkraut zur Sprache. (Alain Finkielkraut, Le Juif imaginaire, Paris 1980.)

39 Bronwyn Winter, Walking the Middle of the Peace Road? The Emergence of JCall in France, in: Modern \& Contemporary France 22 (2014), Heft 1, S. 7-27, hier S. 15.

${ }^{40}$ Mandel, Muslims and Jews, S. 130 und 135.
} 
Unübersehbar und evident wurde diese Problematik jedoch erst um 2000. Seit dem Jahr 2001 waren und sind von Seiten pro-palästinensischer Organisationen immer wieder Boykottforderungen gegen israelische Produkte sowie gegen den Auftritt israelischer KünstlerInnen oder Vorträge von israelischen WissenschaftlerInnen in Frankreich zu vernehmen. ${ }^{41}$ Nachdem 2005 die umstrittene internationale Boykottkampagne BDS (kurz für Boycott, Divestment and Sanctions) ins Leben gerufen worden war, ${ }^{42}$ bildete diese kurze Zeit später auch Strukturen in Frankreich (verstärkt seit dem Gaza-Krieg 2008/09).43 Nimmt man zudem die wesentlichen französischen Pro-PalästinaOrganisationen näher in den Blick, so fällt auf, dass einige unter ihnen erst in den Jahren nach 2000 gegründet wurden. ${ }^{44} \mathrm{Zu}$ den Hauptaktivitäten dieser Vereinigungen zählen neben der Organisation von Demonstrationen auch die Publikation propalästinensischer Artikel und Protestschriften sowie die Organisation von Konferenzen, BenefizKonzerten oder Reisen in die palästinensischen Gebiete, womit sie zum Teil in der Tradition der erwähnten Palästina-Komitees der frühen 1970er-Jahre stehen und an deren politische Agitation anknüpfen. Hinzu kommen ganzjährige oder saisonale Hilfsaktionen vor Ort, insbesondere im Gaza-Streifen sowie im Westjordanland: Das seit 1990 bestehende „Comité de Bienfaisance et de Secours aux Palestiniens“ (CBSP; „Komitee zur Fürsorge und Hilfe für die Palästinenser") etwa gibt auf seiner Homepage an, in palästinensischen Gebieten Aktionen gegen Obdachlosigkeit und Kälte im Winter, für sauberes Trinkwasser oder zum Pflanzen von Olivenbäumen durchzuführen und macht hierzu in Frankreich regelmäßig Spendenaufrufe. ${ }^{45}$ Eine der federführenden französischen Pro-Palästina-Organisationen, die in ihrer jetzigen Form seit 2001 existente „Association France Palestine Solidarité“ (AFPS; „Frankreich Palästina

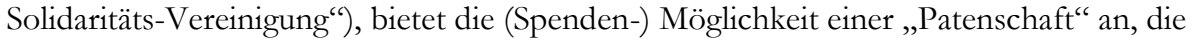
sich sowohl auf palästinensische Kinder (im Westjordanland, in Gaza und im Libanon) ${ }^{46}$ als auch auf palästinensische Gefangene in israelischen Gefängnissen bezieht. ${ }^{47}$ AFPS organisiert zudem regelmäßig Reisen in palästinensische Gebiete (etwa in Form von Sommercamps oder zur Hilfe bei der Olivenernte palästinensischer Bauern). ${ }^{48}$

41 Pascal Markowicz, La délégitimation de l'Etat d'Israël par la campagne de boycott BDS, Paris 2010, S. 1-13.

42 Siehe den Boykottaufruf Palestinian Civil Society Calls for Boycott, Divestment and Sanctions against Israel Until it Complies with International Law and Universal Principles of Human Rights, 9.7.2014,

[http://al-awda.org/pdf/boycotts.pdf], eingesehen 26.10.2018.

43 Winter, Walking the Middle, S. 13.

44 Marc Hecker, Intifada française. De l'importation du conflit israélo-palestinien, Paris 2012, S. 206-210.

45 Siehe etwa die Spendenaufrufe auf Comité de Bienfaisance et de Secours aux Palestiniens, o. D., [http://cbsp.fr], eingesehen 26.10.2018.

46 Association France Palestine Solidarité, o. D., [http://www.france-palestine.org/-Parrainages], eingesehen 26.10.2018.

47 Association France Palestine Solidarité, Parrainer un-e prisonnier-e politique palestinien-ne, 16.7.2012, [http://www.france-palestine.org/Parrainer-un-e-prisonnier-e-politique-palestinien-ne], eingesehen 26.10.2018.

48 Association France Palestine Solidarité, Missions en Palestine, o. D., [http://www.france-palestine.org/-Missionsen-Palestine], eingesehen 26.10.2018. 
Während es sich bei AFPS um eine säkular ausgerichtete Organisation handelt, finden sich auch pro-palästinensische Vereinigungen, die ihre Solidarisierung mit den PalästinenserInnen dezidiert auf den religiösen Bereich ausweiten. ${ }^{49}$ Die Organisation CBSP etwa, welche in Frankreich von der den Muslimbrüdern nahestehenden UOIF („Union des Organisations Islamiques de France“) unterstützt wird und selbst wiederum unter Verdacht steht die Hamas finanziell zu unterstützen, ${ }^{50}$ richtet anlässlich des Fastenmonats Ramadan jährlich an französische MuslimInnen Spendenaufrufe für palästinensische Familien. Gedacht sind die Spenden vor allem für Essenspakete zum „Iftar“ (Fastenbrechen) oder Geschenke für palästinensische Kinder zum „Id al-Fitr““ (Fest zum Ende des Ramadans). Zudem ruft CBSP dazu auf, den vorgeschriebenen „Zakāt al-Fitr" (Almosenabgabe am Ende des Ramadans) den PalästinenserInnen zu widmen..$^{51}$ Neben der Homepage der Organisation und Werbeeinschaltungen auf den muslimischen Online-Nachrichtenseiten SaphirNews und Oumma.com sowie Printwerbung in der muslimischen Monatszeitschrift Salamnews ${ }^{52}$ werden die Spendenaufrufe heute auch über Facebook verbreitet. Ähnliches gilt für die 2009 gegründete konservativislamische Hilfsorganisation „Ligue islamique française pour l'éducation“ (LIFE; „Islamische französische Liga für Bildung“). Während des Ramadans 2015 postete diese über die bekannte islamistische Website Islam \& Info beispielsweise: ${ }^{53}$

„Après la distribution dans les pays d'Afrique, LIFE n'a pas oublié nos frères et sœurs palestiniens. [...]. Il est temps pour nous de nous rapprocher de notre Seigneur en faisant une aumône qui restera inscrite pour le Jour du jugement.“ („Nach der Verteilung in den Ländern Afrikas hat LIFE unsere palästinensischen Brüder und Schwestern nicht vergessen. [...]. Es ist Zeit für uns, uns unserem Herrn zu nähern, indem wir ein Almosen geben, das angerechnet wird für den Tag des (Jüngsten) Gerichts.") Appelliert wird in diesem Posting sowohl an das Mitgefühl französischer MuslimInnen für die „palästinensischen Brüder und Schwestern“ als auch an ihre (religiösen) Pflichten als MuslimInnen an sich. Generell spiegelt die religiöse Durchdringung des Spendenaufrufs

49 Siehe exemplarisch Mérième Alaoui, Solidarité: associations et ONG sur le pont pour Ramadan, in: Salamnews 53 (2015), S. 10-11.

${ }^{50}$ Frank Peter, Die Union des Organisations Islamiques de France und die Tradition der Muslimbrüder im Zeitalter der Integrationspolitik, in: Dietrich Reetz (Hrsg.), Islam in Europa. Religiöses Leben heute. Ein Portrait ausgewählter islamischer Gruppen und Institutionen, Münster 2010, S. 145-169, hier S. 148.

51 Gemäß der Homepage von CBSP gab es für den Ramadan 2015 folgende Spendenmöglichkeiten: 100 Euro um eine Familie während des Ramadan zu unterstützen, 40 Euro für ein Essenspaket, 20 Euro für ein Geschenk an ein palästinensisches Kind und fünf Euro für den persönlichen „Zakāt al-Fitr“. (CBSP, o. D., [http://www.cbsp.fr/dons/index.php?lang=fr], eingesehen 20.6.2015.) Eine wichtige Bedeutung kommt Palästina zudem in den Hilfsaktionen der 1991 gegründeten NGO „Secours Islamique France“ („Islamische Hilfe Frankreich“) zu, welche auf ihrer Webseite ebenfalls zu Spenden aufruft. (Secours Islamique France, o. D., [http://www.secoursislamique.org/component/content/article.html?id=429], eingesehen 26.10.2018.)

52 Siehe Salamnews 53 (2015), S. 5.

${ }^{53}$ Siehe den Werbebanner auf Islam \& Info, Naplouse: LIFE n’a pas oublié nos frères et sœurs palestiniens, 22.6.2015, [http://www.islametinfo.fr/2015/06/22/naplouse-life-na-pas-oublie-nos-freres-et-soeurs-palestiniens], eingesehen 26.10.2018. 
die neuere Tendenz im Bereich der Palästina-Solidarisierungsaktionen wider, sich hierbei dezidiert auf den Islam zu berufen.

In diese Aktionen einzuordnen ist auch die französische Marke „Mecca Cola“. Das Softdrink-Label wurde während des Ramadan 2002 und in Reaktion auf die Zweite Intifada von dem französisch-tunesischen Geschäftsmann Tawfik Mathlouthi im Pariser Vorort Saint Denis nach dem Vorbild der iranischen Marke "ZamZam Cola“ gegründet. ${ }^{54}$ Initiiert wurde das heute weit über die Grenzen Frankreichs hinaus erhältliche Getränk vor allem als Gegenprodukt zu Coca-Cola und zum ,,amerikanischen Hegemonismus“ und gewann insbesondere während des Irakkrieges an Popularität. Ein wesentliches Prinzip von Mecca Cola ist es, zehn Prozent des Profits humanitären Projekten in den palästinensischen Gebieten und weitere zehn Prozent karitativen Organisationen in anderen Ländern zu widmen. ${ }^{55}$ Geprägt zu sein scheint das Firmenoberhaupt Mathlouthi hierbei jedoch nicht nur von antiamerikanischen, sondern auch von antiisraelischen Ressentiments - so ist auf der Webseite von Mecca Cola in Bezug auf die Initiierung der Marke in fehlerhaftem Englisch zu lesen:

„My main Goal also was at that time to point the unfair and unacceptable blind support of America to the Zionist Entity. I couldn't and still can't accept The Policy of Double Standards followed and continuing to be followed by the USA Administration. I Claim for Justice and Human treatment for Palestinians. [...] My Unique reason of strong opposition to these policies is my total rejection of INJUSTICE. I am not driven by hate or rejection of others or any racist feelings. " 56

Zum einen fällt an der Stellungnahme Mathlouthis auf, dass dieser nicht von Israel, sondern, wie für antizionistische Rhetorik charakteristisch, lediglich von einer „Zionist Entity“ spricht. Zum anderen ist die Betonung einer rein humanitären und antirassistischen Intention hinter dem Unternehmen - wohl auch in Abgrenzung zu möglichen Antisemitismusvorwürfen - bezeichnend für pro-palästinensische Initiativen dieser Art. Ähnlich betonte Mathlouthi auch in einem Interview mit der Zeitung Le Parisien im Jahr 2004, er sei „zutiefst antizionistisch“ und gegen den Staat Israel, jedoch ,nicht antijüdisch“. ${ }^{57}$

Wie die verschiedenen Solidaritätskampagnen und Spendenaktionen verdeutlichen, ist die Solidarisierung mit Palästina oftmals auch unmittelbar mit humanitären Aspekten

\footnotetext{
54 Siehe u. a. Bernard Pras/Catherine Vaudour-Lagrâce, Marketing et Islam. Des principes forts et un environnement complexe, in: Revue française de gestion 171 (2007), Heft 2, S. 195-223, hier S. 214; Amel Boubekeur, L'islam est-il soluble dans le Mecca Cola? Marché de la culture islamique et nouveaux supports de religiosité en Occident, in: Maghreb Machrek. 183 (2005), S. 45-65.

55 Siehe u. a. Christin Emrich, Interkulturelles Marketing-Management. Erfolgsstrategien - Konzepte - Analysen, Wiesbaden 2014, S. 16; Frédéric Pons, Mecca Cola à l'assaut du Golfe, in: Libération, 15.8.2003, [http://www.liberation.fr/futurs/2003/08/15/mecca-cola-a-l-assaut-du-golfe_442215], eingesehen 26.10.2018.

56 Tawfik Mathlouthi, The founer [sic] and you, o. D., [https://meccacolagroup.com/the-founer-and-you/], eingesehen 26.10.2018.

${ }^{57}$ „Je suis profondément antisioniste. Je revendique mon anti-Etat d'Israël. Mais je ne suis pas antijuif.“ (Zit. n. Vincent Mongaillard, La saga Mecca-Cola, in: Le Parisien, 16.4.2004, [http://www.leparisien.fr/seine-saint-denis/la-sagamecca-cola-16-04-2004-2004912449.php], eingesehen 26.10.2018.)
} 
verknüpft, deren Bedeutsamkeit hier nicht in Frage gestellt werden soll. Allerdings hat dies wohl auch zur Folge, dass die Israel-Palästina-Frage - unter anderem über gezielte Werbemaßnahmen, Spendenzahlungen oder den bewussten Konsum von Marken wie Mecca Cola - zusätzlich emotional aufgeladen und „personalisiert“ wird. Verbunden ist dies oftmals mit einer religiösen Komponente, insbesondere wenn Palästina-Solidarität in essenzielle Bereiche des Islam, wie die Almosenabgabe Zakāt oder den Ramadan und somit konkret in die religiöse Praxis eingebunden wird. Deutlich wird hieran auch die zentrale, „globalisierte“ Rolle Palästinas für die muslimische „Umma“.

\section{Konklusion}

Wie anhand der Pro-Gaza-Proteste des Sommers 2014 erneut deutlich wurde, werden die Auswirkungen des andauernden Nahostkonflikts in Europa mehr und mehr spürbar - in Frankreich vor allem seit dem Ausbruch der Zweiten Intifada im Jahr 2000, als es zu einem deutlichen Anstieg an antisemitischen Vorfällen kam. Die Proteste formierten sich mit dem offen artikulierten Ziel für die Rechte der Palästinenser und gegen die militärische Reaktion Israels einzutreten. Sie lassen sich schwer - wie die Pro-PalästinaBewegung allgemein - als unmittelbar offen antisemitisch charakterisieren, allerdings positionieren sich viele pro-palästinensische und linksradikale Akteure als offen antizionistisch. Hierbei handelt es sich wiederum um einen Antizionismus, der seit den späten 1960er-Jahren vielfach begleitet ist von Antirassismus, Antikolonialismus, Antiimperialismus und Menschenrechts-Aktivismus, wodurch er paradoxerweise im Namen der „guten Sache“ geäußert wird. ${ }^{8}$ In Anlehnung an Jean Amérys Überlegungen zum „ehrbaren Antisemitismus“59 lässt sich somit die aktuell zu beobachtende PalästinaSolidarität und in manchen Fällen regelrechte "Palästina-Obsession“ als eine Art „ehrbarer“ Antizionismus bezeichnen - im Sinne eines aktivistischen, vordergründig antirassistischen Antizionismus, hinter welchem sich nichtsdestotrotz auch ein mehr oder weniger subtiler Antisemitismus verbergen kann.

Welche Funktionen aber kann - abgesehen von Kanalisierung oder Verschleierung israelfeindlicher oder antisemitischer Ressentiments - ein derartiger Antizionismus erfüllen? Einen Ansatzpunkt hierfür liefert die Historikerin Shulamit Volkov, die ebenfalls in Bezug auf die Palästina-Solidarität der (radikalen) Linken der 1960er und 1970er-Jahre - von „Antizionismus als kulturellem Code“60 spricht: das heißt von Antizionismus als einem Signum kultureller Zugehörigkeit, mit welchem sich gegenüber dem eigenen Umfeld bezeugen lasse, dass man auf der "richtigen Seite“ (der

58 Kloke, Linker Antisemitismus, S. 193-194.

59 Jean Améry, Der ehrbare Antisemitismus. Die Barrikade vereint mit dem Spießer-Stammtisch gegen den Staat der Juden, in: Die Zeit, 25.7.1969, S. 16.

${ }^{60}$ In Anlehnung an ihre These über den Antisemitismus als kulturellen Code. (Shulamit Volkov, Antisemitismus als kultureller Code. Zehn Essays, München 2000.) 
Antiimperialisten, Antikolonialisten, Antirassisten, etc.) stehe. ${ }^{61}$ Die Vermittlung von Zugehörigkeitsgefühlen und eine zugleich nach außen getragene Selbstpositionierung über eine gemeinsam zum Ausdruck gebrachte, „globalisierte“ Palästina-Solidarität scheint heute insbesondere für muslimische Jugendliche in Frankreich und anderen europäischen Ländern von Relevanz zu sein. Dies kann identitätsstiftend wirken und das Gefühl verleihen - gemeinsam mit Gleichgesinnten im eigenen Land und weltweit - auf der moralisch „richtigen“, ,guten“ Seite zu stehen. Zugleich ermöglicht die Identifikation mit den „palästinensischen Brüdern und Schwestern“, das lautstarke Eintreten für deren Rechte auf Demonstrationen und anderswo auch Unzufriedenheit, Enttäuschung, Wut angesichts der eigenen sozialen Situation und Erfahrungen von Rassismus und Islamfeindlichkeit zum Ausdruck zu bringen. Hinzu kommt letztlich, dass die ProPalästina-Bewegung mittlerweile auch eine durchaus gut „vermarktete“ und relativ einfach zu „konsumierende“ Bewegung geworden ist (inklusive zu erstehender propalästinensischer Artikel wie T-Shirts, Fahnen, Schals und Ähnliches, propalästinensischer Rap-Musik ${ }^{62}$ oder Marken wie Mecca Cola etc.). Die Spannweite erstreckt sich hierbei vom politischen bis weit in den muslimisch-religiösen Bereich hinein und ermöglicht so wiederum auf vielfältige Weise, Anschluss zu finden und Zugehörigkeit zu erfahren.

${ }^{61}$ Shulamit Volkov, Readjusting Cultural Codes: Reflections on Anti-Semitism and Anti-Zionism, in: The Journal of Israeli History 25 (2006), Heft 1, S. 51-62, hier S. 60.

62 Ausführlich hierzu siehe Preitschopf, „Umkämpfter Raum“, S. 472-526. 
